REGARDS

SUR L'ECONOMIE ALLEMANDE

BULLETIN ECONOMIQUE DU CRAC

\section{Regards sur l'économie allemande}

Bulletin économique du CIRAC

$72 \mid 2005$

Varia

\title{
Une politique énergétique entre compétitivité et environnement
}

Florence Autret

\section{OpenEdition}

\section{Journals}

Édition électronique

URL : http://journals.openedition.org/rea/281

DOI : 10.4000/rea.281

ISBN : 978-2-8218-0840-9

ISSN : 1965-0787

Éditeur

CIRAC

Édition imprimée

Date de publication : 1 juillet 2005

Pagination : 23-30

ISSN : 1156-8992

Référence électronique

Florence Autret, " Une politique énergétique entre compétitivité et environnement », Regards sur l'économie allemande [En ligne], 72 I juillet 2005, document 3, mis en ligne le 19 juin 2008, consulté le 01 mai 2019. URL : http://journals.openedition.org/rea/281 ; DOI : 10.4000/rea.281 


\title{
Une politique énergétique entre compétitivité et environnement
}

\author{
Florence Autret
}

Depuis quelques années, les prix de l'électricité et du gaz n'ont cessé d'augmenter outre-Rhin. La hausse du coût des matières premières n'est pas seule en cause. Industriels et particuliers subissent les conséquences d'une libéralisation inachevée et de choix radicaux en matière d'approvisionnement en énergie. Peuvent-ils en retour se flatter de performances écologiques supérieures? A voir. La lutte contre le changement climatique est grevée de lourdes hypothèques dues tant au calendrier d'abandon du nucléaire qu'aux modalités de financement des énergies renouvelables. Elle pèse de surcroît indirectement sur la sécurité de l'approvisionnement, dans un contexte d'incertitude peu favorable aux investissements. Après avoir ouvert son premier mandat sous le signe de la "sortie du nucléaire ", le gouvernement SPD/Verts de Gerhard Schröder vient d'opérer un revirement. II cherche maintenant à éviter le triple écueil d'une moindre performance environnementale, de prix élevés et de risques accrus de rupture d'approvisionnement. C'est l'objectif de la nouvelle loi sur l'énergie adoptée le 17 juin et qui instaure une véritable régulation des secteurs électrique et gazier libéralisés il y a 7 ans.

Toute politique énergétique est tiraillée entre trois impératifs : la sécurité d'approvisionnement (Versorgungssicherheit), la viabilité économique (Wirtschaftlichkeit) et le respect de l'environnement (Umweltverträglichkeit). Pour avoir voulu concilier tous ces objectifs, l'Allemagne court le risque de n'en satisfaire aucun; mais il se pourrait aussi qu'elle parvienne à un compromis optimal. A cet égard, la mise en œuvre de la loi du 17 juin et l'évolution du contexte international seront décisives. Mais une chose est d'ores et déjà certaine : d'ici 20 ans, le paysage énergétique allemand sera profondément différent.

La décennie 2020 est celle de toutes les échéances. Le calendrier de sortie du nucléaire négocié en 2001 prévoit la déconnection de la dernière tranche en 2021. A cet horizon, les industriels estiment que près de la moitié des capacités de production actuelles auront dû être renouvelées (y compris mais pas principalement du fait de l'abandon du nucléaire). C'est dans la première moitié de la décennie aussi que l'électricité éolienne, aujourd'hui largement subventionnée, devrait, selon les experts, commencer à être rentable. A ce moment-là, si les objectifs du gouvernement sont atteints, les énergies renouvelables devraient assurer un cinquième de l'approvisionnement électrique du pays. Tel est le scénario optimiste présenté par la coalition SPD/Verts : des centrales nucléaires remplacées par des éoliennes et des centrales au gaz, un parc de centrales et un réseau modernisés, des émissions de $\mathrm{CO}_{2}$ maîtrisées. Si l'ambition est limpide, le chemin pour y parvenir apparaît incertain et semé d'embûches.

La situation actuelle se situe à mille lieues de ce paysage idéal. Les consommateurs, petits et grands, se plaignent de payer leur énergie nettement plus cher que les autres Européens ; les grands opérateurs, à la fois producteurs et exploitants de réseau, répugnent à investir pour cause "d'insécurité juridique » et menacent le pays de black-out ; le calendrier d'abandon du nucléaire est plus contesté que jamais ; et enfin, Berlin se retrouve dans le collimateur de la Commission européenne qui stigmatise son manque d'empressement à garantir une

2005-2025: un chemin semé d'embûches 
Quotas 2005/07 : 1497 millions de tonnes de $\mathrm{CO}_{2} /$ an, mais...

véritable concurrence sur un marché officiellement libéralisé depuis 1998. Jamais, en somme, les dilemmes inhérents à toute politique énergétique n'ont été aussi manifestes. Le futur paysage énergétique dépend de multiples facteurs plus ou moins prévisibles. Mais s'il en est un fermement défini, c'est bien celui de la lutte contre le changement climatique.

En février 2005, 1849 installations industrielles allemandes se sont vu allouer une enveloppe de droits à émettre du $\mathrm{CO}_{2}$ correspondant à 1497 millions de tonnes par an, pour la période 2005-2007. Les centrales électriques, raffineries, usines de ciment et autres verreries concernées figurant sur cette liste doivent donc dès cette année ajuster leurs émissions sur leurs quotas. En cas de dépassement, elles devront acquérir sur le marché les quotas manquants. En cas d'excédent, elles pourront valoriser leurs quotas en les revendant sur le marché. En février 2005, le prix de la tonne de $\mathrm{CO}_{2}$ négociée à la bourse de l'électricité de Leipzig (EEX) se situait autour de $8,5 €$.

\section{Le système européen d'échanges de quotas d'émission de $\mathrm{CO}_{2}$}

L'origine de la directive de 2003 créant le système européen d'échange de quotas de $\mathrm{CO}_{2}$ remonte au protocole de Kyoto de 1997. En le ratifiant, l'UE s'engage à réduire ses émissions de gaz à effet de serre de $8 \%$ d'ici 2012 par rapport aux émissions constatées en 1990. Pour atteindre son objectif, la Commission s'est penchée prioritairement sur les gaz à effet de serre produits par l'industrie, l'une des trois principales sources avec le transport aérien et automobile et l'habitat. Deux options étaient ouvertes : la taxation ou la "marchandisation » des émissions. N'ayant pas de compétences fiscales, la Commission s'est rangée à l'idée d'un mécanisme marchand qui avait d'ailleurs la préférence des industriels. Régi par la directive 2003/87/EC, le système européen d'échange de droits d'émissions de gaz à effet de serre concerne environ 12000 installations industrielles représentant le moitié des émissions européennes. Les sites entrant dans ce périmètre sont les installations de combustion, raffineries d'hydrocarbures, cokeries, installations sidérurgiques et aciéries, ainsi que les usines fabriquant du ciment, du verre, de la chaux, de la brique, de la céramique, de la pâte à papier et du papier.

Entre les différents modèles de marché existants, Bruxelles a opté pour celui appelé « cap and trade » : un plafond (cap) absolu des droits d'émission dans la limite duquel les acteurs peuvent s'échanger les droits (trade). En abaissant le plafond, les autorités publiques renchérissent le coût du $\mathrm{CO}_{2}$. Limités en quantité, les quotas d'émission sont alloués gratuitement aux industriels, lesquels doivent 'rendre' à la fin de chaque année aux autorités publiques des quotas équivalents à leurs émissions annuelles. S'ils disposent de quotas en excédent, ils peuvent les vendre sur le marché. Si au contraire leurs émissions ont excédé leur 'capital', ils doivent acheter des quotas supplémentaires, faute de quoi ils sont passibles d'une amende dissuasive (40€ par quota non restitué). La première 'restitution', pour l'année 2005, aura lieu en avril 2006.

La directive confie aux Etats membres la tâche d'allouer des quotas d'émissions correspondant au burden sharing entre Etats membres, autrement dit à la contribution de chaque Etat à l'effort que l'UE s'est engagée à produire. Le volume de quotas que chaque Etat peut distribuer est lui-même soumis au contrôle de la Commission. Elle guide les Etats dans l'élaboration des "plans nationaux d'allocation » (PNA) de quotas pour une première période de 3 ans (2005-2007). Une nouvelle allocation est prévue fin 2007 pour les cinq ans suivants, jusqu'à l'échéance de Kyoto, à savoir 2012. Cette procédure a donné lieu, comme on l'imagine, à un lobbying intense des industriels dans tous les pays européens. Premier sujet de débat : la définition du périmètre des installations concernées (la directive laisse une marge d'interprétation : fallait-il ou non, par exemple, inclure les centrales électriques réservées à l'alimentation d'un site industriel lui-même exclu du champ d'application ? Et si oui, à partir de quelle capacité ?). Second sujet : la période de référence qui servirait de base de calcul pour l'attribution des quotas et la fixation d'un objectif d'émissions. Troisième sujet : les conditions de 'transfert' des certificats d'un site à l'autre, dans le même pays ou dans deux pays différents, au sein d'un même groupe.

L'Allemagne fut, en 2004, l'un des rares pays à présenter son PNA dans les délais fixés par la directive (Nationaler Allokationsplan établi par le ministère de l'Environnement, de la Protection de la Nature et de la Sécurité nucléaire, 31 mars 2004). Ce plan a été approuvé par la Commission européenne par une décision du 7 juillet 2004, à quelques réserves près. Mais toutes les questions soulevées par le système européen n'ont pas été résolues et, à bien des égards, la première période (2005-2007) devrait faire office de test grandeur nature. Si la Commission européenne a estimé que le PNA allemand était en règle, certains industriels allemands qui s'estimaient inéquitablement traités ont annoncé qu'ils pourraient ester en justice. Le Royaume-Uni, à l'inverse, a souhaité revoir à la hausse le total des émissions de $\mathrm{CO}_{2}$ alloué à ses industriels. Devant le refus de la Commission européenne, Londres a annoncé qu'elle contesterait cette décision devant la Cour de Justice européenne, tout en acceptant de rester dans le cadre validé par la Commission en attendant la décision de Luxembourg.

... une faible contrainte pour l'Allemagne
Alors que l'Allemagne participait ainsi au lancement du «système européen d'échanges de quotas d'émission de $\mathrm{CO}_{2}$ ", le protocole de Kyoto en vertu duquel ce système avait été conçu par l'UE entrait enfin en vigueur, 7 ans après sa signature, grâce à l'adhésion in extremis de la Russie. Rappelons que le protocole de Kyoto vise à réduire de $5 \%$ d'ici 2012 par rapport à 1990 les émissions de gaz à effet de serre (GES) constatées sur la planète. Son entrée en vigueur était conditionnée à la signature d'un groupe d'Etats représentant ensemble au moins $55 \%$ des émissions de la planète, seuil que la ratification par le parlement russe en décembre 2004 a permis de dépasser. Le fait que l'allocation des quotas se soit faite de manière relativement aisée n'est pas sans lien 
avec la manière dont les engagements européens de Kyoto ont été répartis entre pays membres de l'Union et avec les obligations qui en ont découlé pour l'Allemagne. Au moins pour la première période (2005-2007), la contrainte est relativement limitée. Cela s'explique aisément par les performances déjà enregistrées depuis 1990, année de référence du protocole, retenue également en règle générale pour le calcul des quotas dans le système européen.

Dans le cadre de Kyoto, l'objectif de réduction des GES assigné à I'UE est de $8 \%$ en 2012 par rapport à 1990. Cette réduction a été ensuite répartie entre les Etats membres. Plus important émetteur de GES de I'Union, l'Allemagne s'est ainsi vu assigner un objectif impressionnant de réduction de ses GES de $21 \%$. Dès 2002, elle n'était néanmoins pas loin de l'avoir atteint puisque les émissions avaient déjà diminué de $19 \%$ par rapport à l'année de référence. Cette 'surperformance' s'explique en réalité largement par la chute rapide des émissions dans les nouveaux Länder dans les années qui ont suivi la réunification. La transformation de l'appareil industriel a d'ailleurs produit les mêmes effets dans les pays d'Europe centrale nouvellement entrés dans l'UE, relâchant pour eux aussi la contrainte de Kyoto.

Pour la période 2008-2012, en revanche, I'Union européenne a prévu d'accroître l'incitation à la baisse des émissions en réduisant plus significativement la quantité de quotas en circulation. Cet objectif inquiète les producteurs d'électricité allemands et constitue une réelle contrainte en termes de choix énergétiques. Dans cette perspective, il est intéressant de se pencher sur les facteurs d'évolution des émissions.
1990-2002 : les émissions de GES ont baissé de $19 \%$ en RFA

Evolution des émissions de $\mathrm{CO}_{2}$ par secteur (en millions de tonnes)

\begin{tabular}{|lrrrrr|}
\hline & $\mathbf{1 9 9 1}$ & $\mathbf{1 9 9 4}$ & $\mathbf{1 9 9 7}$ & $\mathbf{2 0 0 0}$ & $\mathbf{2 0 0 3}$ \\
Secteur énergétique & 427,9 & 387,5 & 364,2 & 364 & 385,1 \\
Mines, industrie manufacturière & 146,5 & 128,2 & 123,6 & 116,3 & 107,3 \\
Transport & 161,5 & 168,9 & 173,1 & 178,3 & 166,5 \\
$\quad$ dont automobile & 154,0 & 160,9 & 165,9 & $\mathbf{1 7 1 , 1}$ & 159,8 \\
Foyers & 131,5 & 128,4 & 138,4 & 116,8 & 122,4 \\
Commerce, services, petite industrie & 86,3 & 67,4 & 68,8 & 59,2 & 60,3 \\
\hline Total des émissions induites & & & & & \\
par la consommation d'énergie & 953,6 & 880,4 & 868,1 & 834,6 & 841,7 \\
Total des émissions induites & & & & & \\
par les processus industriels & 23,3 & 25,3 & 25,4 & 25,5 & 23,7 \\
Total & $\mathbf{9 7 6 , 9}$ & $\mathbf{9 0 5 , 7}$ & $\mathbf{8 9 3 , 5}$ & $\mathbf{8 6 0 , 1}$ & $\mathbf{8 6 5 , 4}$ \\
\hline
\end{tabular}

Source : DIW Wochenbericht n9/2005

L'institut DIW a publié en 2005 une étude détaillée de l'évolution et surtout de l'origine des émissions de $\mathrm{CO}_{2}$ depuis 1990. Cinq facteurs expliquent l'évolution des émissions de $\mathrm{CO}_{2}$ : la démographie, les revenus (autrement dit, la croissance), l'intensité énergétique (c'est-à-dire l'évolution du ratio émissions de $\mathrm{CO}_{2}$ / produit intérieur brut), l'Energiemix (la répartition entre sources de production d'énergie produisant ou ne produisant pas d'émissions) et enfin l'évolution du contenu en $\mathrm{CO}_{2}$ des sources d'énergie fossiles utilisées (la combustion du gaz dégageant par exemple moins de $\mathrm{CO}_{2}$ que celle du charbon). Sur les deux périodes analysées : 1990-1997 et 1997-2004, l'évolution des trois derniers facteurs, sources de baisse des émissions, fait plus que compenser les facteurs de hausse (démographie et croissance). Néanmoins, leur impact diminue très nettement sur la seconde période par rapport à la première. En particulier, la composante Energiemix n'a d'impact que marginal, montrant que l'évolution du panier d'approvisionnement en énergies primaires n'est plus une source significative de réduction des émissions. En d'autres termes, depuis 1997, les centrales électriques ne contribuent pas à la baisse des émissions.

Les données de l'institut iwd (Cologne) corroborent ce résultat. En 2004, les émissions de $\mathrm{CO}_{2}$ d'origine énergétique étaient inférieures de 162 millions de
La baisse des émissions depuis 1990 est due essentiellement...

... à la modernisation des centrales énergétiques de l'est 
$200000 €$ la journée d'inactivité d'une centrale nucléaire

Le prix de l'électricité, nouvelle variable d'ajustement

tonnes à leur niveau de 1990. Néanmoins $82 \%$ (133 millions de tonnes) de cette réduction ont été réalisés pendant la période 1990-1995. Les bonnes performances allemandes sont donc exclusivement imputables aux améliorations de l'outil énergétique est-allemand. Entre 1990 et 1995, les émissions y ont baissé de $44 \%$ tandis qu'elles stagnaient pratiquement $(+2 \%)$ dans les anciens Länder. Passée cette phase d'adaptation des installations est-allemandes, la baisse est devenue à peine perceptible. Entre 1995 et 2004, les émissions n'ont baissé que de 3,3 \% (-29 millions t) dans l'ensemble de la RFA.

Dans le contexte du resserrement de la contrainte exercée sur les industriels par le système européen d'échange de quotas, les électriciens doivent donc fournir un effort particulier en dépit du plan de fermeture des centrales nucléaires. Selon une récente étude d'Ixix (groupe Caisse d'épargne), les électriciens allemands devront tous abaisser leurs émissions de 29 millions de tonnes pour la seconde période, contre 13 millions de tonnes pour la première. Or la valorisation des émissions de $\mathrm{CO}_{2}$ a un effet non négligeable sur les rapports de compétitivité entre les différentes sources d'énergie. Prenons à titre d'exemple une centrale nucléaire restée 'improductive' un jour de plus que prévu pour cause, par exemple, de prolongation de sa révision. Ce jour-là, $24000 \mathrm{MWh}$ devront être produits dans une autre centrale, par exemple une centrale au charbon, laquelle émet 1,1 million de tonnes de $\mathrm{CO}_{2}$ par $\mathrm{MWh}$. Le montant des émissions induites par la substitution entre les deux centrales serait donc de 25000 tonnes. Valorisées au prix du marché, soit 8,5€, cela représente un surcoût d'environ $200000 €$ uniquement à cause du $\mathrm{CO}_{2}$.

Comment donc réduire les émissions tout en réduisant la production nucléaire sans faire exploser les prix de l'électricité ? Tel est le dilemme auquel est confrontée l'Allemagne. Le gouvernement y a apporté une première réponse en misant sur le développement des énergies renouvelables. Ce choix a fait du pays un précurseur en la matière. Mais il n'est pas dénué de risques en termes de sécurité d'approvisionnement, et il pose un problème de compétitivité puisque les capacités supplémentaires d'électricité produite à partir d'énergies renouvelables ne peuvent pas à ce jour être financées aux conditions du marché. Le prix de l'électricité est donc apparu comme la véritable variable d'ajustement du marché électrique dans la période récente, les subventions aux renouvelables et l'internalisation du 'coût du $\mathrm{CO}_{2}$ ' étant financées non pas par le contribuable mais par les consommateurs.

Sources d'énergie utilisées pour la production d'électricité (en PJ)

\begin{tabular}{|l|r|r|r|rr|r|r|}
\hline & 1991 & 1995 & 1999 & \multicolumn{2}{|c|}{2003} & $2003 / 1991$ & $2030{ }^{*}$ \\
Charbon & 1354 & 1332 & 1272 & 1298 & $(24 \%)$ & $-4 \%$ & $-8 \%$ \\
Lignite & 1678 & 1455 & 1328 & 1539 & $(28 \%)$ & $-8 \%$ & $-13 \%$ \\
Fuel + autres énerg. fossiles & 188 & 142 & 117 & 164 & $(3 \%)$ & $-13 \%$ \\
Gaz & 416 & 433 & 504 & 510 & $(9 \%)$ & $+23 \%$ & $3 \%$ \\
Hydraulique et éolien & 85 & 105 & 94 & 158 & $(3 \%)$ & $+86 \%$ & $26 \%$ \\
Nucléaire & 1608 & 1681 & 1855 & 1802 & $(33 \%)$ & $+12 \%$ & $0 \%$ \\
\hline Total & 5329 & 5148 & 5170 & 5471 & $(100 \%)$ & $+3 \%$ & $100 \%$ \\
\hline
\end{tabular}

Sources : BMWA 2004/05, sauf $\left(^{*}\right)$ : projections extraites du rapport EWI/Prognos (doc. $n^{\circ} 545$ ).

Objectif de la politique de soutien aux énergies renouvelables

$20 \%$ d'électricité 'propre' en 2020
Dans toute l'Europe, et surtout en Allemagne, les énergies renouvelables jouissent d'un cadre économique et juridique particulièrement avantageux. La loi Erneubare Energiegesetz (EEG), révisée en août 2004, assure à l'électricité produite à partir d'elles à la fois une priorité absolue de reprise sur le réseau et un prix de rachat assuré. Le résultat de cette politique volontariste et généreuse se fait d'ores et déjà sentir. Le parc éolien allemand a été décuplé en 10 ans. II était en 2004 de loin le plus important d'Europe, avec 16600 MW répartis en 16500 installations (soit environ la moitié des installations de toute l'Europe). Les conditions de reprise de l'électricité ainsi produite ont été conçues par la loi EEG pour atteindre l'objectif de $12,5 \%$ de l'électricité produite à partir de renou- 
velables en 2010, puis $20 \%$ en 2020. On notera qu'en 2002, l'électricité produite à partir d'éoliennes était de 16,8 TWh (0,3 en 1992), contre 26 TWh pour les centrales hydrauliques, autre principale source d'électricité 'propre', 294 pour le charbon et le lignite, 54 pour le gaz et 165 pour le nucléaire.

Si l'essor de l'éolien a été assuré jusqu'à présent par l'implantation de parcs situés principalement dans les régions côtières du nord, les objectifs inscrits de I'EEG ne pourront être atteints que grâce aux fermes offshore comme il en existe déjà dans les eaux territoriales danoises, britanniques, irlandaises ou suédoises. Ces installations offshore, parce qu'elles bénéficient de conditions climatiques plus favorables, sont seules susceptibles de garantir à terme la compétitivité de l'électricité éolienne. Compte tenu des projets actuellement à l'étude, l'Allemagne pourrait disposer d'une capacité offshore supplémentaire de 2000 à $3000 \mathrm{MW}$ dès 2010. Les premiers projets pilote doivent être testés d'ici 2007. A l'horizon 2020-2030, le parc éolien représenterait une capacité de 20000 à $25000 \mathrm{MW}$, soit l'équivalent de la capacité actuelle du parc des centrales au gaz ou de celui des centrales nucléaires.

\section{Evolution des quantités d'électricité produites et subventionnées} dans le cadre de la loi EEG (en GWh)

\begin{tabular}{|ccc|}
\hline & Electricité produite à partir d'énergies renouvelables & dont éolien \\
2000 & 13855 & 7550 \\
2002 & 24963 & 15856 \\
2004 & 37097 & 24158 \\
\hline 2006 & 48829 & 33325 \\
2008 & 61467 & 43721 \\
2010 & 80800 & 61312 \\
\hline
\end{tabular}

Source des données et projections: Verband der Netzbetreiber (VDN), in EEG-Mittelfristprognose 2000-2010. NB : le trait en pointillés indique le moment où on bascule de la donnée réelle à la projection.

Mais l'essor de l'éolien ne va pas sans difficultés, principalement l'inadaptation du réseau de transport et son financement grâce à des prix de rachat très supérieurs à ceux du marché. Ce second point est à l'origine de la modification de la loi EEG en août 2004 : elle répartit entre les 4 principaux exploitants de réseau (E.On, RWE, EnBW, Vattenfall) le surcoût qui était jusqu'alors majoritairement supporté par E.On, en position dominante dans les régions du nord où l'essentiel de l'électricité éolienne entre sur le réseau. La question des capacités du réseau est plus délicate. Pour y répondre, le gouvernement a commandé en 2004 une étude à l'Agence fédérale de l'énergie (Deutsche Energie Agentur, dena) laquelle a associé l'ensemble des acteurs du marché à son travail.

Ses principaux résultats, publiés en février 2005 et largement commentés dans la presse, sont particulièrement instructifs. Premièrement, ils montrent un important besoin de mise à niveau du réseau qui tient notamment au fait que près de la moitié (45\%) de l'électricité éolienne n'est pas utilisée localement mais revendue et distribuée sur le réseau public. Le développement de l'éolien renforce les capacités de production dans le Nord alors que la demande se situe plutôt au Sud. II appellerait ainsi la création de $850 \mathrm{~km}$ de lignes à haute tension et la rénovation de $400 \mathrm{~km}$, pour un coût total de 1,1 milliard $€$ d'ici 2015 . Deuxièmement, le développement de l'éolien appelle une adaptation du reste du parc électrique, en particulier une augmentation des capacités en pointe au détriment des capacités en base, afin de palier les aléas de la production éolienne tout en permettant que cette dernière soit écoulée prioritairement sur le réseau. La production en base a en effet pour but de satisfaire une demande en électricité relativement constante sur une année, par opposition à la production en pointe. Un moyen de production en base est donc une installation qui fonctionne environ deux tiers du temps, voire plus, alors qu'un moyen de production en pointe fonctionne de manière beaucoup plus occasionnelle et est donc plus de nature à remédier à une éventuelle baisse de la capacité éolienne.
Priorité à l'éolien offshore

Mais une étude de la dena révèle 2 sérieux handicaps :

... l'inadaptation du réseau de transport... 
... et la charge financière des subventions
La flambée des prix pétroliers contrecarre la sortie du nucléaire

Forte hausse des prix au consommateur

Une loi pour achever la libéralisation du marché de l'électricité
Le développement de centrales au gaz, particulièrement flexibles, apparaît ainsi comme un nécessaire corollaire de l'éolien, à l'inverse du nucléaire, production en base par excellence.

L'étude de la dena fournit enfin une évaluation du « surcoût » induit par le développement du parc offshore. Le prix du MW offshore s'établit autour de 1,8 million $€$, y compris l'installation et la connexion au réseau continental, soit $80 \%$ de plus qu'une installation terrestre. Ainsi, l'objectif de $2000 \mathrm{MW}$ offshore en 2010 supposerait un investissement de 3,6 milliards $€$. Pour finir, le rapport dena estime le surcoût induit par la loi sur le soutien aux énergies renouvelables à $30 \%$ (du prix du KWh) à l'horizon 2015 (pour une capacité éolienne de 16000 $\mathrm{MW}$ ) par rapport à un scénario sans soutien aux renouvelables. Cette publication, qu'on peut lire comme une opération vérité sur le coût de la sortie du nucléaire et de la préservation du climat, a connu un retentissement d'autant plus grand qu'elle intervenait en pleine discussion entre les industriels, le gouvernement et le parlement, sur la nouvelle loi sur l'énergie.

\section{Les enjeux de la nouvelle loi sur l'énergie}

En matière de politique énergétique, le gouvernement fédéral disposait, au début de son premier mandat, d'une bonne marge de manœuvre qui tenait moins à la cohérence de sa politique qu'à un environnement favorable. Les prix des matières premières étaient au plus bas, de même que les coûts marginaux de production. Performantes et amorties ou en passe de l'être, les infrastructures de production et de transport étaient au plus fort de leur rentabilité. Sept ans plus tard, la situation s'est totalement retournée : le prix du baril de pétrole est passé de 20 à $50 \$$ entre fin 2001 et 2004 et, surtout, l'industrie aborde un nouveau cycle où les besoins d'investissement s'annoncent considérables. Pour la production d'électricité, le renouvellement nécessaire des capacités est estimé à $40000 \mathrm{MW}$ à l'horizon 2020 et à $60000 \mathrm{MW}$ à l'horizon 2025, soit pratiquement la moitié des capacités actuelles (120 $000 \mathrm{MW})$. Les investissements correspondants sont de l'ordre de 10 milliards $€$ par millier de MW. Cet effort est d'autant plus nécessaire qu'il participe de l'impératif de réduction des émissions de $\mathrm{CO}_{2}$. La modernisation des centrales thermiques devrait permettre de réduire les émissions de $\mathrm{CO}_{2}$ de 46 millions de tonnes.

Parallèlement, alors que Berlin avait promis que l'ouverture du marché à la concurrence se traduirait pour le consommateur par une baisse des prix, ces derniers ont très fortement augmenté depuis 5 ans. Un tableau comparatif publié en janvier 2005 par le quotidien Frankfurter Allgemeine Zeitung montre que ces hausses atteignent un sommet de $45 \%$ pour les clients d'EnBW dans la région de Stuttgart, et se situent entre $10 \%$ et $30 \%$ dans de nombreuses régions ou villes ( $31 \%$ à Düsseldorf, $27 \%$ à Brême, par exemple). Le kWh allemand est le plus cher d'Europe $(0,858 €)$, juste derrière l'Italie $(0,92 €)$ et loin devant la France (prix moyen $0,598 €$; source : Eurostat). Ces prix élevés ne reflètent pas seulement la hausse du prix des matières premières, mais aussi l'échec relatif de l'introduction de la concurrence dans la foulée des directives européennes de libéralisation.

La nouvelle loi sur l'énergie (Zweites Gesetz zur Neuregulierung der Energiewirtschaft), votée le 17 juin 2005 et entrée en vigueur le $1^{\text {er }}$ juillet, doit précisément achever la libéralisation. Depuis sept ans, l'Allemagne a laissé se concentrer son marché, sous la surveillance, il est vrai, de l'Office fédéral des Cartels, mais sans contrôle efficace de ce paramètre essentiel qu'est l'accès au réseau. Au terme de cette évolution, le marché se caractérise par une structure à la fois oligopolistique en amont (autour de E.On, RWE, EnBW et Vattenfall) et décentralisée vers l'aval (1700 exploitants de réseau entretenant généralement des liens capitalistiques avec l'un ou l'autre des 4 'grands'), et par des prix élevés. $A$ posteriori, on peut estimer qu'il n'était pas possible d'avancer sur tous les fronts 
à la fois : négociation de la sortie du nucléaire, subvention à l'éolien et maîtrise du coût de l'énergie. Toujours est-il que c'est seulement en 2004, sous la pression de la Commission européenne, que le gouvernement a entrepris de doter le marché allemand d'outils de régulation adaptés. Sceptique sur le système dit " négocié " d'accès au réseau privilégié par l'Allemagne en 1998, et alerté par les concurrents des groupes allemands impuissants à pénétrer ce marché, la Commission a plusieurs fois enjoint Berlin d'assurer une réelle concurrence sur son marché et de créer une autorité de régulation sectorielle qui se saisisse du problème de l'accès au réseau au plus tard le $1^{\mathrm{er}}$ juillet... 2004.

Le texte long et technique adopté en juin 2005 comporte deux dispositions majeures. II soumet d'une part le secteur de l'énergie au contrôle d'une autorité sectorielle alors que celui-ci relevait jusqu'alors de l'Office fédéral des Cartels. La nouvelle Agence fédérale des réseaux de l'électricité, du gaz, des télécommunications, de la poste et des chemins de fer (Bundesnetzagentur für Elektrizität, Gas, Telekommunikation, Post und Eisenbahn), est en fait une extension de l'Autorité de régulation des télécommunications (RegTP) et restera présidée par le juriste Matthias Kurth, nommé à sa tête en 2001. Elle doit employer à terme 180 personnes, principalement des juristes et des économistes.

L'autre innovation majeure de ce projet de loi réside dans l'instauration d'un mécanisme de contrôle des prix de transport. Le gouvernement ne le nomme pas ainsi, et pourtant c'est bien de cela qu'il s'agit. Aux termes d'un compromis trouvé avec les Länder les plus exigeants en matière de concurrence (la Hesse et la Bavière, qui sont aussi des bastions de la CDU/CSU), la future autorité exercera un contrôle ex ante sur les prix de transport. La loi prévoit en effet un mécanisme dit d'incitation (Anreize), en réalité un prix plafond garanti fixé ex ante. L'exploitant est ainsi incité à abaisser ses coûts pour maximiser sa marge, garantie, sous réserve qu'il maintienne son réseau en bon état. C'est pourquoi le prix garanti est assorti d'exigences en matière de mise à niveau du réseau. Comme on l'imagine, les critères selon lesquels pourra être établi ce prix font déjà débat. Si la loi fixe un cadre, ce mécanisme d'incitation reste à négocier avec les exploitants et les utilisateurs. Cela sera une des tâches essentielles de la nouvelle autorité qui disposera d'un an après son installation, prévue cet été, pour établir ces tarifs. Cependant, dès sa création et jusqu'à la mise ne place du système, elle est chargée d'exercer un contrôle ex-post sur les augmentations de tarifs pratiquées par les exploitants. La loi édicte le principe général de Nettosubstanzerhaltung, selon lequel la rémunération de l'exploitant doit couvrir le maintien à niveau des installations, avec ce que cela suppose de travaux de maintenance et d'entretien, plus une rémunération du capital immobilisé. La rémunération du capital investi demandée par les exploitants est de 6,4\% pour le réseau électrique, et de $7,8 \%$ pour le réseau gazier. Le gouvernement compte avec ce système concilier deux objectifs : contrôler la flambée des prix d'accès au réseau et assurer la concurrence, d'un côté, tout en encourageant l'investissement, de l'autre.

Examinée en commission de conciliation le 29 avril puis à nouveau le 15 juin, la nouvelle loi $E W G$ a été définitivement adoptée le 17 juin. Mais le gouvernement a dû faire preuve de souplesse pour obtenir que la loi soit votée à temps pour entrer en vigueur le $1^{\mathrm{er}}$ juillet 2005 , exactement un an après l'adoption du projet en conseil des ministres. Pris en tenaille entre les intérêts des exploitants, ceux des Länder et ceux des consommateurs, il a multiplié réunions et consultations tout au long des derniers mois à la recherche d'un compromis. L'opposition CDU/CSU, qui a fait de ce projet le double emblème de son attachement à la concurrence et de la défense des Länder et des consommateurs, a obtenu plusieurs amendements. Le contrôle concurrentiel sort renforcé du débat parlementaire. Les prix de transport en particulier devront faire l'objet d'une validation systématique par l'autorité fédérale des réseaux, alors que le Bundestag ne demandait qu'un contrôle sur les augmentations intervenues après le $1^{\text {er }}$ août 2004. Mais l'enjeu réel de la loi était bien de doter le gouvernement fédéral d'un
Création d'une autorité de régulation sectorielle

Vers un contrôle des prix de transport

Des projets compromis par les élections de septembre 2005 ? 
moyen de peser sur les prix d'utilisation des réseaux, donc les investissements d'infrastructure. Ce souci, ajouté à la pression exercée par Bruxelles pour mettre en place une véritable régulation, était visiblement suffisamment partagé par l'opposition pour qu'on aboutisse à un accord. Si bien qu'une éventuelle alternance au Bundestag à l'automne prochain ne remettra pas en cause ce nouveau cadre juridique.

L'AlLEMAGNe N'AVAIT PAS D'AUTRE CHoIX que de revoir son système de régulation. Elle poursuit ainsi son chemin vers un mode de régulation original qui n'est ni le monopole à prix réglementés à la française, ni le système marchand britannique, mais une sorte de "cartel régulé ". Si la nouvelle loi accroît le rôle de l'autorité fédérale, elle ne règle pas tout pour autant et ménage une place importante à la consultation des exploitants et producteurs, publics ou privés, grands industriels et consommateurs. Pour reprendre les termes des directives européennes, l'Allemagne se situe à mi-chemin entre une système réglementé et un système négocié.

Mais cette nouvelle étape ne la dispense pas de répondre à la question du prix qu'elle est prête à payer pour atteindre ses objectifs environnementaux. Or pour beaucoup, ce prix est trop élevé et appelle le relâchement d'au moins une contrainte, à savoir la sortie du nucléaire. Depuis l'automne 2004, on ne compte plus les études et prises de position demandant, sinon un ajournement, du moins un aménagement de l'échéancier de sortie du nucléaire. La durée de vie moyenne d'une centrale est actuellement de 32 ans alors que, selon les experts, elle peut être prolongée jusqu'à 50 ans dans des conditions financières favorables puisque les coûts fixes baissent avec l'allongement de la durée d'utilisation. De la Fédération des Chambres de commerce (DIHT) à celle de I'Industrie (BDI) en passant par celle des Electriciens (Verband der Deutschen Elektrizätswerke, VDEW) ou de l'Electrotechnique et de l'Electronique (Zentralverband Elektrotechnik- und Elektronikindustrie, ZVEI), le prolongement de la durée de vie des centrales est présenté comme le seul moyen de respecter les engagements en termes d'émissions de gaz à effet de serre en en limitant l'impact sur la compétitivité. On peut en conclure qu'une possible alternance politique se traduira par un étalement du calendrier de fermeture des centrales, sans modifier radicalement les orientations dessinées par le gouvernement Schröder.

\section{Indications bibliographiques}

\footnotetext{
AUtRET F., "L'avenir de la filière nucléaire civile », in Bourgeols I. (dir.), Allemagne 2001. Regards sur une économie en mutation, CIRAC, Levallois, 2001

BOURGEOIS I., "Même régulation pour les télécoms et l'énergie ? ", Regards sur l'économie allemande, $n^{\circ} 63 / 03$, et "L'Allemagne face à la crise pétrolière : l'écotaxe et la politique énergétique à l'épreuve ", in BouRGEOIS I. (dir.), Allemagne 2001. Regards sur une économie en mutation, CIRAC, Levallois, 2001

Courivaud H., " Le secteur de l'électricité à l'heure de la libéralisation ", in BouRGEoIs I. (dir.), Allemagne 2001. Regards sur une économie en mutation, CIRAC, Levallois, 2001 DENA, Energiewirtschaftliche Planung für die Netzintegration von Windenergie in Deutschland an Land und Offshore bis zum Jahr 2020 (www.deutsche-energie-agentur.de) Dossier spécial énergie, ifo Schnelldienst, $\mathrm{n}^{\circ} 4 / 05$

EWI/Prognos, Die Entwicklung der Energiemärkte bis zum Jahr 2030, Doc. n`545

KEMFERT C., "Strommarkt in Europa : zwischen Liberalisierung und Klimaschutz ", DIW Wochenbericht, $\mathrm{n}^{\circ} 31 / 04$

"Klimaschutz. Kyoto kommt », iwd, 10-02-2005

MichaELOWA A., "Großzügige Versorgung der Großemittenten mit $\mathrm{CO}_{2}$-Emissionsrechten ", HWWA-Forum, Wirtschaftsdienst 2004

"Strom wird in Deutschland immer teurer », Frankfurter Allgemeine Zeitung, 19-01- 2005

ZIESING H.-J., « Stagnation der Kohlendioxid-Emissionen in Deutschland im Jahre 2004 », DIW Wochenbericht, $\mathrm{n}^{\circ} \mathrm{9} / 05$
} 